\title{
Transcriptional co-regulator RIP140: An important mediator of the inflammatory response and its associated diseases (Review)
}

\author{
ZHU-JUN YI ${ }^{1,2}$, JIAN-PING GONG ${ }^{1,2}$ and WEI ZHANG ${ }^{3}$ \\ ${ }^{1}$ Department of Hepatobiliary Surgery; ${ }^{2}$ Key Laboratory of Hepatobiliary Surgery in Chongqing, \\ The Second Affiliated Hospital of Chongqing Medical University, Chongqing, Sichuan 400010; \\ ${ }^{3}$ Department of Hepatobiliary Surgery, The People's Hospital of Jianyang, Jianyang, Sichuan 641400, P.R. China
}

Received January 9, 2016; Accepted February 13, 2017

DOI: $10.3892 / \mathrm{mmr} .2017 .6683$

\begin{abstract}
The inflammatory response is a physiological process that is essential for maintaining homeostasis of the immune system. Inflammation is classified into acute inflammation and chronic inflammation, both of which pose a risk to human health. However, specific regulatory mechanisms of the inflammatory response remain to be elucidated. Receptor interacting protein (RIP) 140 is a nuclear receptor that affects an extensive array of biological and pathological processes in the body, including energy metabolism, inflammation and tumorigenesis. RIP140-mediated macrophage polarization is important in regulating the inflammatory response. Overexpression of RIP140 in macrophages results in M1-like polarization and expansion during the inflammatory response. Conversely, decreased expression of RIP140 in macrophages reduces the number of M1-like macrophages and increases the number of alternatively polarized cells, which collectively promote endotoxin tolerance (ET) and relieve inflammation. This review summarizes the role of RIP140 in acute and chronic inflammatory diseases, with a focus on insulin resistance, atherosclerosis, sepsis and ET.
\end{abstract}

\section{Contents}

1. Introduction

2. Macrophage-mediated insulin resistance and obesity resulting from RIP40 in adipose tissues

3. Role of RIP140 in the development of atherosclerosis

4. Role of RIP140 in ET

5. Conclusions and future directions

Correspondence to: Professor Wei Zhang, Department of Hepatobiliary Surgery, The People's Hospital of Jianyang, 180 Yixueyuan Road, Jianyang, Sichuan 641400, P.R. China E-mail: zw68229@sina.com

Key words: receptor interacting protein 140, insulin resistance, atherosclerosis, sepsis, endotoxin tolerance

\section{Introduction}

Nuclear receptors regulate cell function by controlling the expression of specific gene networks. They are essential for the regulation of energy metabolism and immune homeostasis (1). Receptor interacting protein (RIP) 140 is a ligand-dependent nuclear receptor that controls the transcription of target genes in various tissues, including adipose, skeletal muscle, cardiac muscle, liver and tumor tissues (1-3). RIP140 functions as a metabolic switch that regulates numerous metabolic pathways involved in defensive functions via interaction with transcription factors (4). As a co-repressor, RIP140 facilitates high-fat diet-induced obesity, increases energy expenditure and induces insulin resistance $(5,6)$. In addition, RIP140 affects tumorigenesis and tumor metastasis via the E2F transcription factor and wingless-type mouse mammary tumor virus/adenomatous polyposis coli/ $\beta$-catenin signaling pathways (7-9). As a co-activator, RIP140 activates nuclear factor $\kappa \mathrm{B}(\mathrm{NF}-\kappa \mathrm{B})$ and promotes the expression of proinflammatory cytokines, including tumor necrosis factor (TNF)- $\alpha$, interleukin (IL)-1 $\beta$ and IL-6 in immune cells, particularly in macrophages (10). Furthermore, NF-kB-mediated degradation of the RIP140 co-activator may induce endotoxin tolerance (ET) (11). Type 2 diabetes and cardiovascular diseases are the result of insulin resistance and atherosclerosis, respectively. These metabolic disorders are macrophage-mediated chronic inflammatory diseases (1). Sepsis is a systemic inflammatory response syndrome (SIRS) and is a common clinical disease. ET may significantly alleviate the inflammatory response and reduce the mortality rates of individuals with sepsis and septic shock (12). The present brief review summarizes the role of RIP140 in the macrophage-mediated inflammatory response involving insulin resistance, atherosclerosis, sepsis and ET.

2. Macrophage-mediated insulin resistance and obesity resulting from RIP40 in adipose tissues

Insulin resistance and obesity are important factors in the development of metabolic syndromes, and pose a significant threat to human health. It has previously been demonstrated that the inflammatory response of macrophages in adipose 


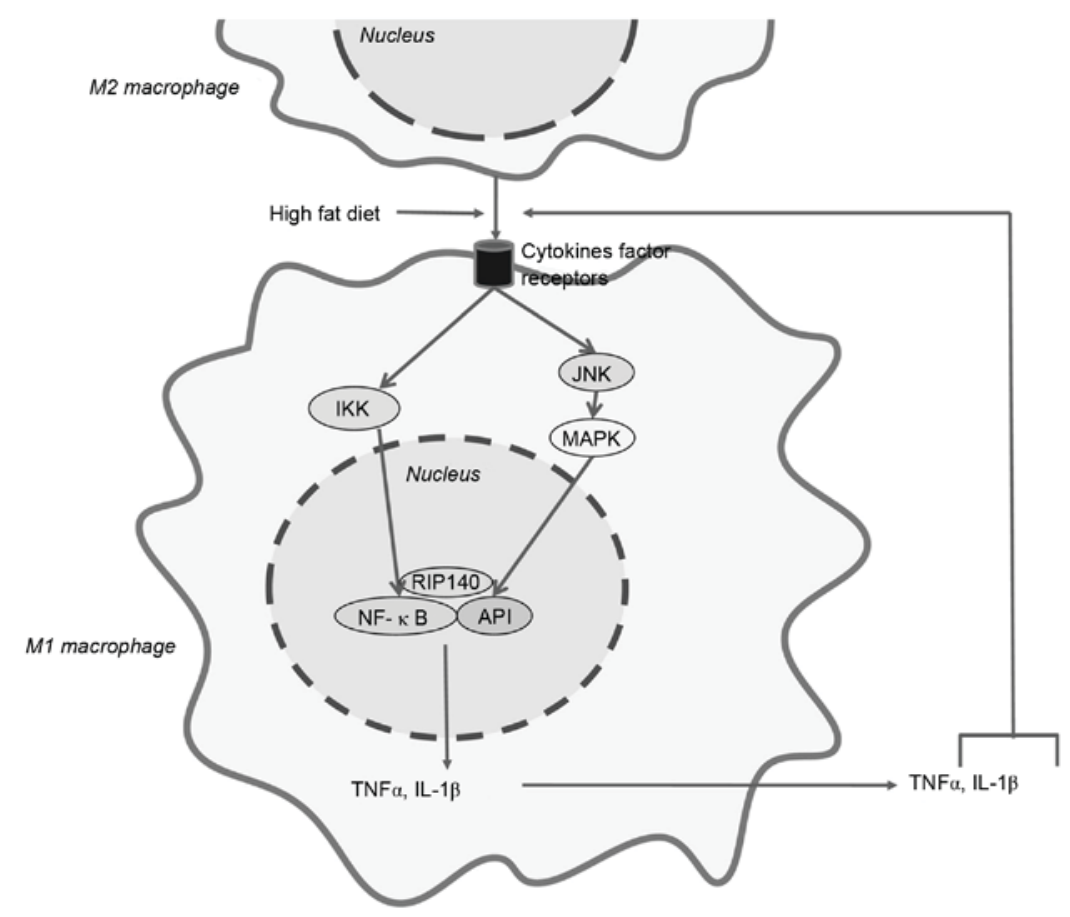

Figure 1. A high-fat diet promotes M1-like polarization of macrophages in adipose tissues. High fat diet-induced JNK1/MAPK/RIP140/AP1 and IKK are involved in the activation of NF- $\mathrm{KB}$. Activated NF- $\mathrm{KB}$ promotes the expression of pro-inflammatory genes, which facilitates the subsequent production of TNF- $\alpha$ and IL-1 $\beta$. High levels of TNF- $\alpha$ and IL-1 $\beta$ form part of a positive feedback system that induces the M1-like polarization of macrophages. JNK, Jun $\mathrm{N}$-terminal kinase; MAPK, mitogen-activated-protein-kinase; RIP140, receptor interacting protein 140; AP1, activator protein-1; IKK, inhibitor of NF- $\mathrm{B}$

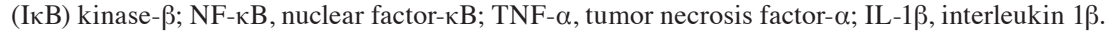

tissues appears to result in insulin resistance in the skeletal muscle (13).

Insulin resistance is a hallmark of type 2 diabetes and metabolic syndrome. A high level of free fatty acids (FFAs) in the blood plasma has been identified as an important mediator of obesity-associated insulin resistance in the skeletal muscle (14). Adipocytes demonstrate the ability to synthesize and store a large quantity of triglycerides (TG). Adipocytes may additionally hydrolyze and release TGs as FFAs and glycerol during fasting. These properties of adipocytes maintain a dynamic equilibrium between FFA release into the circulation and FFA uptake and oxidation by the peripheral tissues, primarily in skeletal muscles. Kelley et al (15) demonstrated that elevated levels of circulating FFAs may lead to insulin resistance in the peripheral tissues of animals and humans.

Adipose tissue may be classified as white adipose tissue (WAT) or brown adipose tissue (BAT). WAT is primarily observed in adults, whereas BAT is primarily observed in infants (16-18). Numerous studies have demonstrated that macrophages in adipose tissue represent $\sim 50 \%$ of the total number of cells in high-fat diet-induced obese patients. However, this value decreases to $\sim 5-10 \%$ in people of a healthy weight (19-21). In mice fed on a high-fat diet, the level of RIP140 expression in macrophages is elevated, which promotes macrophages to undergo M1-like polarization. In addition, a high-fat diet enhances macrophage recruitment to WAT and facilitates insulin resistance $(22,23)$. By contrast, knockout of RIP140 in monocytes or macrophages decreases the level of RIP140 expression in differentiated macrophages and promotes an anti-inflammatory phenotype via M2-like polarization, which increases insulin sensitivity (22-24).
Macrophage-mediated chronic inflammation in adipose tissue promotes the release of FFAs from adipocytes, which is associated with the development of insulin resistance in skeletal muscles. Adipocytes and macrophages secrete a significant quantity of monocyte chemoattractant protein-1 (MCP-1)/chemokine (C-C motif) ligand-2 (CCL2), TNF- $\alpha$ and IL-1, which induces an inflammatory response in adipose tissue (25). Transgenic overexpression of MCP-1 in adipocytes enhances macrophage infiltration in adipose tissues, which subsequently promotes the inflammatory response and induces insulin resistance in skeletal muscles. By contrast, knockout of the MCP-1 receptor chemotactic cytokine receptor 2 in adipocytes reduces the inflammatory response in adipose tissues and increases insulin sensitivity in skeletal muscles. In normal physiology, MCP-1/CCL2, TNF $\alpha$ and IL-1 mediate the inflammatory response in adipose tissues, which is important for metabolic regulation in adipocytes. However, when adipocytes and macrophages secrete a large quantity of these cytokines, two significant effects on adipocyte function occur; an increase in lipolysis and decrease in TG synthesis $(26,27)$. These actions result in increased levels of circulating TGs and FFAs. The excess of circulating TG and FFAs leads to their accumulation in skeletal muscles, which subsequently disrupts mitochondrial oxidative phosphorylation and insulin-mediated glucose transport, thereby facilitating insulin resistance in skeletal muscle.

In people of a healthy weight, $\sim 5 \%$ of total adipose tissue cells are macrophages, and these demonstrate an anti-inflammatory M2-like polarization state. By contrast, a large number of M1-like macrophages accumulate in the adipose 


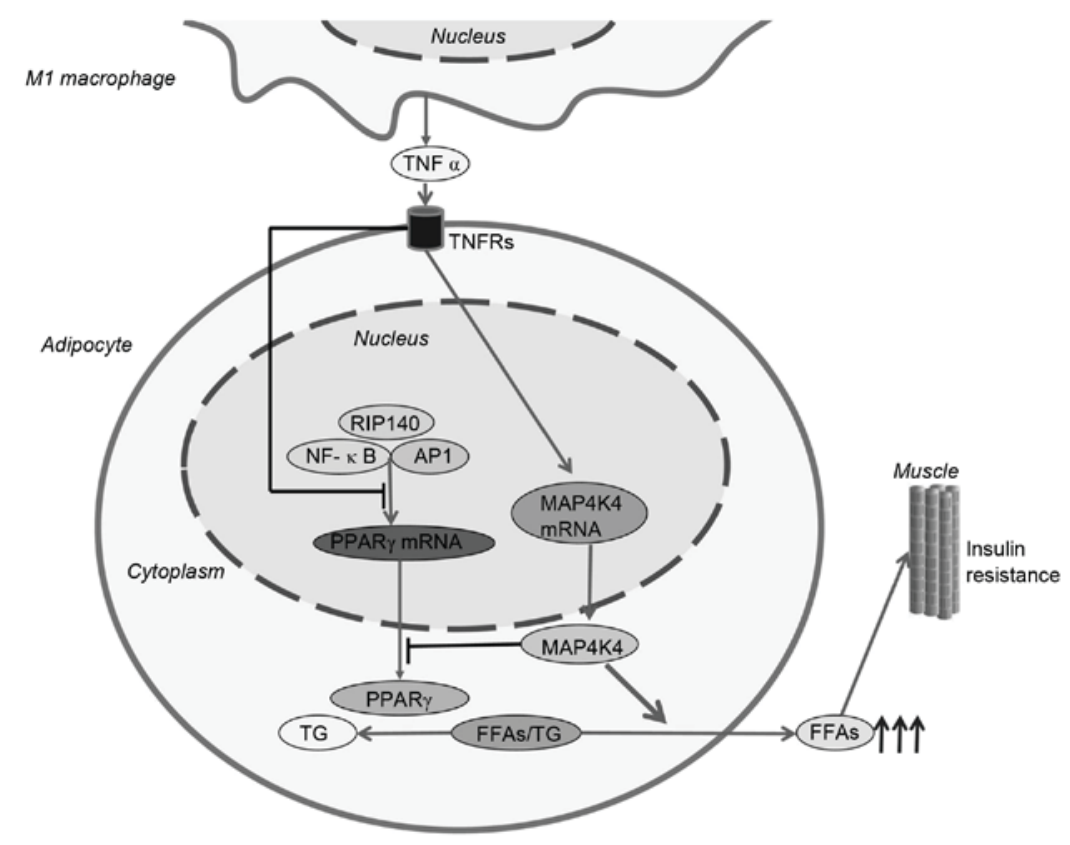

Figure 2. Overexpression of TNF- $\alpha$ in M1-like macrophages activates RIP140/NF-kB/AP1 signaling pathways in adipocytes and inhibits the expression of PPAR $\gamma$ mRNA at the transcriptional level. TNF- $\alpha$ promotes the expression of MAP4K4. MAP4K4 inhibits PPAR $\gamma$ at the level of translation and enhances

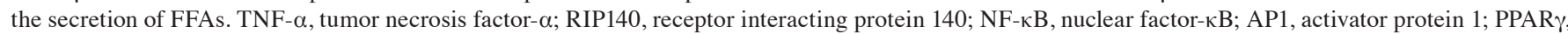
peroxisome proliferator-activated receptor $\gamma$; MAP4K4; mitogen-activated-protein 4-kinase-4; TNFR, tumor necrosis factor receptor; TG, triglycerides; FFA, free fatty acids.

tissues of patients with obesity (24). Numerous studies have revealed that RIP140 is important for the development of the M1-like polarization characteristic of macrophages $(22,23)$. Decreasing the level of RIP140 in macrophages reduced the number of M1-like macrophages and increased the number of M2-like macrophages (21). M1-like macrophages promote the expression of pro-inflammatory cytokines, including TNF $\alpha$ and IL-1 $\beta$, which decrease WAT browning and enhance high-fat diet-induced insulin resistance (11). RIP140 activates the NF- $\kappa \mathrm{B}$ pathway, which is the central step for the production of TNF $\alpha$ and IL-1 $\beta$ in macrophages. The Jun $\mathrm{N}$-terminal kinase-mitogen-activated-protein 4-kinase-4 (MAP4K4)-activator protein-1 (AP1) and inhibitor of $\mathrm{NF}-\kappa \mathrm{B}$ $(\mathrm{I} \kappa \mathrm{B})$ kinase- $\beta-\mathrm{NF}-\kappa \mathrm{B}$-dependent signaling pathways are additionally important for the activation of NF- $\kappa$ B (Fig. 1) (28-31). High levels of TNF- $\alpha$ and IL-1 $\beta$ interact with adipocytes to promote the production of FFAs (32). The specific mechanisms underlying this interaction remain to be elucidated; however the evidence suggests that TNF- $\alpha$ and IL-1 $\beta$ may induce peroxisome proliferator-activated receptor $\gamma$ (PPAR $\gamma)$ at the transcriptional and translational levels (33). PPAR $\gamma$ is a member of the nuclear receptor family, is a key transcriptional regulator of the uptake and storage of $\mathrm{TG}$, and promotes adipogenesis (34). At the transcriptional level, TNF- $\alpha$ inhibits the expression of PPAR $\gamma$ mRNA. This is due, in part, to TNF- $\alpha$-mediated activation of the RIP140, NF- $\kappa$ B and AP1 signaling pathways. At the translational level, mitogen-activated protein kinase (MAPK) is a negative regulator of PPAR $\gamma$ protein expression. MAPK functions to decrease the expression of PPAR $\gamma$ and promote the release of TG and FFAs. High levels of TG and FFAs in the circulation facilitate insulin resistance in skeletal muscles (Fig. 2) (35-37). The specific mechanisms underlying PPAR $\gamma$-mediated regulation of fatty acid esterification, TG synthesis and hydrolysis remains to be elucidated. TNF- $\alpha$ and IL-1 $\beta$ promote M1-like polarization of macrophages and induce insulin resistance (Fig. 1) (38). The mechanism underlying IL-1 $\beta$-impaired insulin sensitivity in adipose tissues may be associated with the inhibition of insulin signal transduction; however this remains to be fully elucidated (39).

\section{Role of RIP140 in the development of atherosclerosis}

Atherosclerosis is an early-stage lesion of coronary artery disease and myocardial infarction, which poses a serious threat to human health. It is known that hypercholesterolemia is an essential component for the development of various cardiovascular diseases, particularly atherosclerosis. During hypercholesterolemia, accumulating cholesterol leads to the formation of an atheroma plaque. Numerous inflammatory cells, such as macrophages, are recruited and may lead to chronic inflammation (40). The primary function of macrophages is to scavenge excess peripheral cholesterol. However, macrophages differentiate into foam cells with long-term high levels of cholesterol in blood. The emergence of foam cells signifies the development of atherosclerosis (41). Under physiological conditions, high-density lipoprotein (HDL) and low-density lipoprotein (LDL) maintain the dynamic balance of cholesterol metabolism. HDL transports excess cellular cholesterol from peripheral tissues to the liver, which subsequently decreases the formation of atherosclerotic plaques. By contrast, LDL transports cholesterol from the liver to peripheral tissues and facilitates the formation of atherosclerotic plaques $(40,41)$. Numerous studies have demonstrated that ATP-binding membrane cassette transporter member A1 (ABCA1) and ATP-binding cassette subfamily $\mathrm{G}$ member 1 


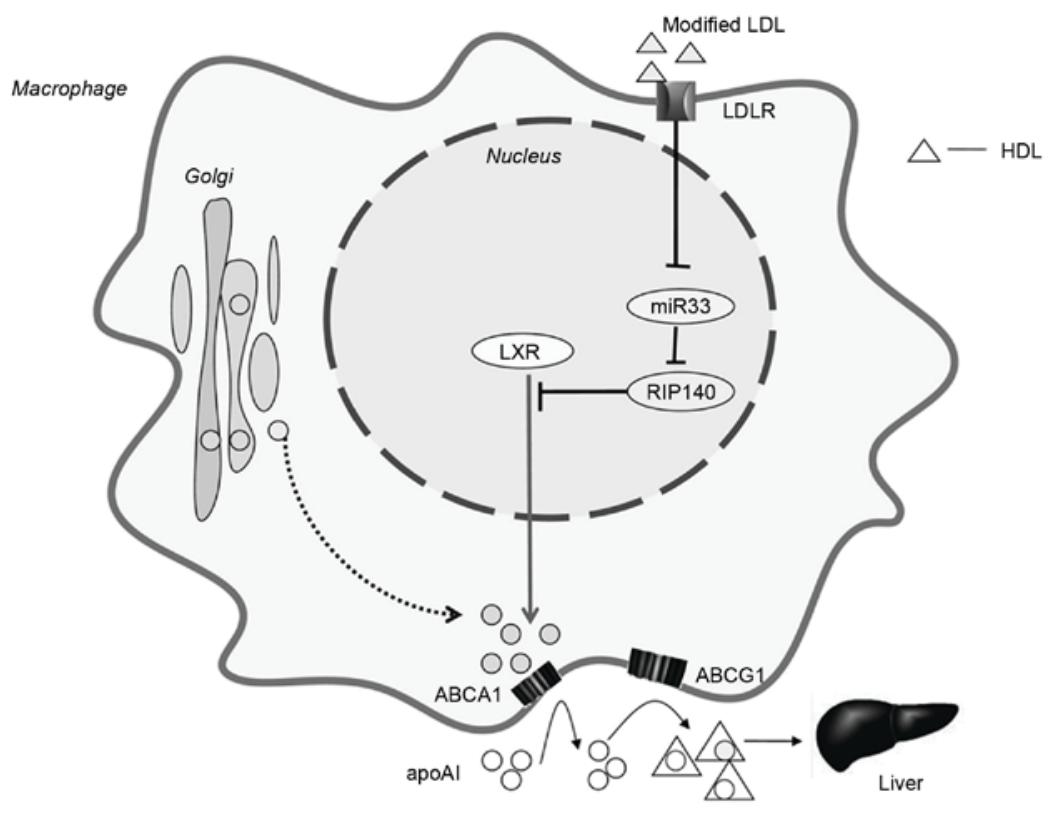

Figure 3. RIP140 inhibits the efflux of cholesterol in macrophages. Modification of LDL elevates RIP140 and reduces miR33 expression levels in macrophages. Overexpression of RIP140 in macrophages inhibits LXR-mediated ABCA1 and ABCG1 signaling pathways, which inhibits transport of cholesterol across the membrane. During these processes, ABCA1, ABCG1, apoAI and HDL collectively mediate cholesterol efflux. RIP140, receptor interacting protein 140; LDL, low-density lipoprotein; miR33, microRNA 33; LXR, liver X receptor; ABCA1, ATP-binding membrane cassette transporter member A1; ABCG1, ATP-binding cassette subfamily G member 1; apoAI, apolipoprotein AI; HDL, high-density lipoprotein; LDLR, low-density lipoprotein receptor.

(ABCG1) interact with HDL to increase cholesterol efflux and promote cholesterol transportation to its lipid-depleted receptor apolipoprotein $\mathrm{AI}$ (apoAI), which protects against the formation of foam cells and the development of atherosclerosis $(42,43)$. The specific mechanism remains to be elucidated; however it is apparent that RIP140 contributes to foam cell formation and atherosclerosis by regulating cholesterol homeostasis in macrophages. RIP140 suppresses the expression of ABCA1 and ABCG1 in macrophages and thus inhibits the efflux of cholesterol. In an in vivo study, the short hairpin RNA-mediated knockdown of RIP140 in peritoneal macrophages of mice resulted in the increased expression of ABCA1 and ABCG1, which increased cholesterol efflux (44). Conversely, overexpression of RIP140 in macrophages reduced the expression of $\mathrm{ABCA} 1$ and $\mathrm{ABCG} 1$, and increased the accumulation of cholesterol $(44,45)$.

Liver X receptor (LXR) is a nuclear receptor that promotes cholesterol efflux by directly regulating the expression of ABCA1 and ABCG1. RIP140 is a co-repressor of LXR, which inhibits LXR-mediated ABCA1 and ABCG1 signaling pathways (46-48). However, it has been demonstrated that activation of hepatic LXR may induce lipogenesis and lead to hepatic steatosis. The enhancement of peripheral LXR activity without affecting hepatic LXR is of primary concern (47). In addition, cholesterol-responsive microRNA (miR)-33 is a negative regulator of RIP140 expression in macrophages, by directly binding to a highly conserved sequence in the 3 -untranslated region of RIP140 mRNA (49,50). A previous study demonstrated that cholesterol upregulates RIP140 expression by repressing miR-33 expression (49). In addition, miR-758, miR-10b, miR-144, miR-27 and miR-26 directly repress ABCA1/ABCG1 and negatively regulate cholesterol efflux in macrophages (51). Ultimately, the potential of RIP140 as a target for the treatment of atherosclerosis is evident (Fig. 3) (52).

\section{Role of RIP140 in ET}

SIRS is a very common clinical condition, with severe sepsis and septic shock associated with a high mortality rate. Despite the use of numerous types of antibiotics, the mortality rate of patients with severe sepsis and septic shock remain high at $\sim 30 \%$ (53). During infection, a large number of bacteria permeate the blood, thus inducing an innate immune response. Lipopolysaccharide (LPS) endotoxin is located in the bacterial cell wall. A number of bacteria are killed during the innate immune response, which leads to the release of LPS into the blood. LPS activates numerous types of inflammatory cells, such as macrophages and monocytes, to promote the production of inflammatory cytokines, thus resulting in sepsis and septic shock $(54,55)$. A prototypical inflammatory pathway has been established from numerous years of research. The LPS-mediated inflammatory response is induced by activating toll-like receptor 4 (TLR4) located on an inflammatory cell membrane, which facilitates TLR4-mediated activation of the downstream myeloid differentiation primary response gene 88 (MyD88). IL-1 receptor-associated kinase (IRAK) 4 molecules on MyD88 facilitate IRAK1 phosphorylation and activation. MyD88 and IRAK activate the NF- $\mathrm{BB}$ signaling pathway to facilitate the transcription of pro-inflammatory genes, which subsequently leads to the production of further inflammatory cytokines, including TNFa, IL-1 $\beta$ and IL-6 (Fig. 4A) (56-59). Previous studies have indicated that inflammatory cells repeatedly stimulated with low-dose LPS develop ET $(11,12)$. Inflammatory cytokines produced by these inflammatory cells are subsequently reduced, which reduces the incidence and mortality rate of sepsis and septic shock (53). A more detailed understanding of the mechanisms underlying ET is important for the development of novel therapeutic treatments for sepsis and septic shock. It has previously been demonstrated that loss of 


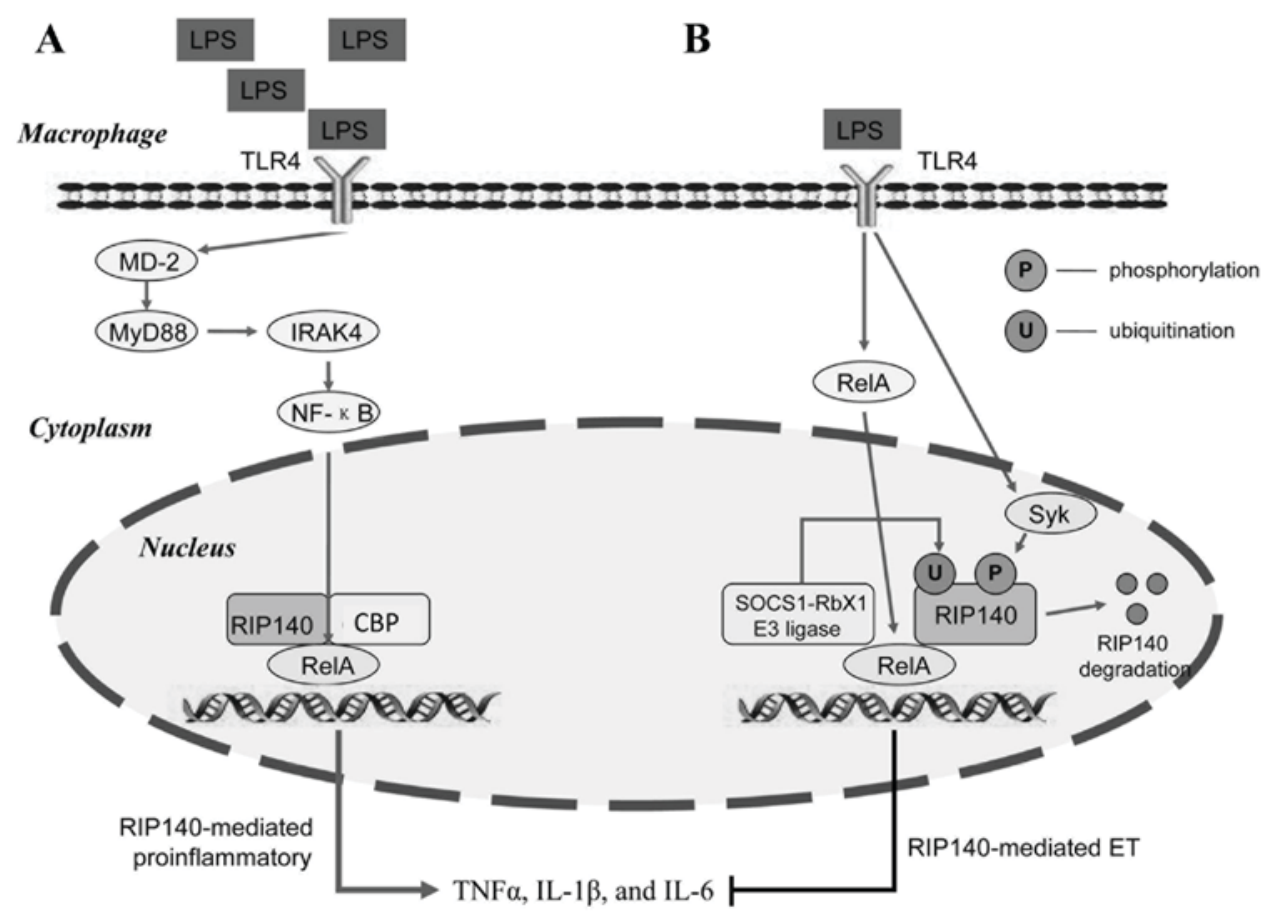

Figure 4. (A) RIP140-mediated pro-inflammatory response in macrophages. LPS mediates the inflammatory response by activating TLR4 on the cell membrane.

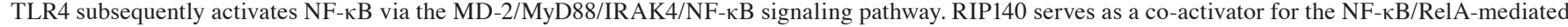
inflammatory response by recruiting CBP to promote the expression of TLR4-induced pro-inflammatory cytokines, including TNF $\alpha$, IL-1 $\beta$ and IL-6. (B) Degradation of RIP140 meditates LPS-induced ET. A low dose of LPS activates NF-кB/RelA by interacting with TLR4. RIP140 interacts with RelA to co-activate SOCS1-Rbx1 transcriptional activity, which results in the recruitment of SOCS1-Rbx1 E3 ligase. SOCS1 and Rbx1 may promote RIP140 ubiquitination, which is required for RIP140 degradation. In addition, LPS activates Syk-mediated phosphorylation of RIP140, which leads to its ubiquitination and induces ET. RelA-mediated SOCS1-Rbx1 E3 ligase recruitment and Syk-mediated tyrosine phosphorylation are necessary for LPS-induced RIP140 degradation. Degradation of RIP140 in macrophages reduces the production of inflammatory factors including TNF $\alpha$, IL-1 $\beta$ and IL-6. RIP140, receptor

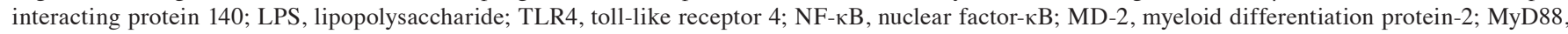
myeloid differentiation primary response gene 88; IRAK4, IL-1 receptor-associated kinase 4; CBP, cAMP response element binding protein-binding protein; TNF- $\alpha$, tumor necrosis factor- $\alpha$; IL, interleukin; ET, endotoxin tolerance; SOCS1, suppressor of cytokine signaling 1; Rbx1, RING-box protein 1; Syk, spleen tyrosine kinase.

RelA binding, histone modifications and chromatin remodeling are essential factors for the development of ET (60). However, the specific mechanisms underlying these alterations remain to be elucidated. Previous studies have demonstrated that RIP140 functions as a co-activator of the NF- $\kappa \mathrm{B} / \mathrm{RelA}$-mediated inflammatory response by recruiting cAMP response element binding protein-binding protein (CBP) to promote the expression of TLR4-induced pro-inflammatory cytokines, including TNF $\alpha$, IL-1 $\beta$ and IL-6 (Fig. 4A) $(10,11)$. It has previously been demonstrated that RIP140 degradation is critical for LPS-induced ET (11). The suppressor of cytokine signaling 1 (SOCS1)-RING-box protein 1 (Rbx1) has been revealed to interact with RelA and promote RelA degradation in cell nuclei. RIP140 interacts with RelA to co-activate SOCS1-Rbx1 transcriptional activity. In addition, RIP140 interacts with RelA to mediate the recruitment of SOCS1-Rbx1 E3 ligase. SOCS1 and Rbx1 may promote RIP140 ubiquitination, which is required for RIP140 degradation (11). In addition, LPS activates spleen tyrosine kinase (Syk)-mediated phosphorylation of RIP140 on Tyr364, Tyr418 and Tyr436 residues, thus facilitating its ubiquitination and inducing ET (11). RelA-mediated SOCS1-Rbx1 E3 ligase recruitment and Syk-mediated tyrosine phosphorylation are necessary for LPS-induced RIP140 degradation (Fig. 4B) (11). Interferon- $\gamma($ IFN- $\gamma$ ) activates macrophages to amplify the inflammatory response and promote the expression of pro-inflammatory cytokines that abrogate ET (61). Pre-treatment of macrophages with IFN- $\gamma$ inhibits RIP140 degradation, and overexpression of non-degradable RIP140 effectively diminishes LPS-induced ET in vitro and in vivo (11).

\section{Conclusions and future directions}

Type 2 diabetes, cardiovascular diseases, sepsis and septic shock are the most common diseases with major societal implications $(11,13,41)$. These diseases are all macrophage-meditated inflammatory diseases; Sepsis and septic shock may additionally be classified as acute inflammatory diseases. However, chronic inflammatory diseases, including diabetes and cardiovascular diseases may be more harmful than acute diseases, and it is important to investigate the underlying molecular mechanisms involved. RIP140 functions as a nuclear receptor and co-regulator that is involved in insulin resistance, atherosclerosis, sepsis and ET $(13,41)$.

In mice with high-fat diet-induced obesity, macrophages with high expression levels of RIP140 accumulate in adipose tissues (23). Elevation of RIP140 induces M1 polarization in macrophages and facilitates the release of FFAs, which subsequently results in insulin resistance of skeletal muscles (15,35-37). In mice with hyperlipidemia, RIP140 suppresses the expression of ABCA1 and ABCG1 in macrophages (47). Inhibition of the efflux of cholesterol then contributes to foam cell formation and atherosclerosis (47). In acute inflammatory diseases, 
RIP140 serves as a co-activator for the NF- $\kappa$ B/RelA-mediated inflammatory response by recruiting CBP to promote expression of TLR4-induced pro-inflammatory cytokines, including TNF- $\alpha$, IL-1 $\beta$ and IL-6 (11). Furthermore, inflammatory cells that are repeatedly stimulated with low-dose LPS develop ET (12). The underlying mechanism involves degradation of RIP140 via interaction with RelA, the SOCS1-Rbx1 E3 ligase and Syk, and the subsequent reduction of the inflammatory cytokine expression, which contributes to ET (11). Despite ongoing research regarding the role of RIP140 in inflammatory diseases, further studies are required to determine the underlying molecular mechanisms involved in ET (1-4). Verifying the clinical relevance of RIP140 as a prognostic marker may be beneficial for the diagnosis and treatment of these diseases. In addition, intestinal LSP-mediated excessive activation of hepatic macrophages, such as Kupffer cells (KCs), may be important in liver ischemia-reperfusion injury following liver transplantation (62). Investigating whether RIP140 may reduce liver ischemia-reperfusion injury during liver transplantation via inducing ET of KCs is a focus of current research, which may facilitate an improved understanding of the role of RIP140 in the inflammatory response.

\section{References}

1. Rosell M, Jones MC and Parker MG: Role of nuclear receptor corepressor RIP140 in metabolic syndrome. Biochim Biophys Acta 1812: 919-928, 2011.

2. White R, Morganstein D, Christian M, Seth A, Herzog B and Parker MG: Role of RIP140 in metabolic tissues: Connections to disease. FEBS Lett 582: 39-45, 2008.

3. Fritah A, Christian M and Parker MG: The metabolic coregulator RIP140: An update. Am J Physiol Endocrinol Metab 299: E335-E340, 2010.

4. Chung HT: RIP140, a Janus metabolic switch involved in defense functions. Cell Mol Immunol 10: 7-9, 2013.

5. Ho PC, Chuang YS, Hung CH and Wei LN: Cytoplasmic receptor-interacting protein 140 (RIP140) interacts with perilipin to regulate lipolysis. Cell Signal 23: 1396-1403, 2011.

6. Powelka AM, Seth A, Virbasius JV, Kiskinis E, Nicoloro SM, Guilherme A, Tang X, Straubhaar J, Cherniack AD, Parker MG and Czech MP: Suppression of oxidative metabolism and mitochondrial biogenesis by the transcriptional corepressor RIP140 in mouse adipocytes. J Clin Invest 116: 125-136, 2006.

7. Docquier A, Harmand PO, Fritsch S, Chanrion M, Darbon JM and Cavaillès V: The transcriptional coregulator RIP140 represses E2F1 activity and discriminates breast cancer subtypes. Clin Cancer Res 16: 2959-2970, 2010.

8. Lapierre M, Bonnet S, Bascoul-Mollevi C, Ait-Arsa I, Jalaguier S, Del Rio M, Plateroti M, Roepman P, Ychou M, Pannequin J, et al: RIP140 increases APC expression and controls intestinal homeostasis and tumorigenesis. J Clin Invest 124: 1899-1913, 2014.

9. Zhang D, Wang Y, Dai Y, Wang J, Suo T, Pan H, Liu H, Shen S and Liu H: Downregulation of RIP140 in hepatocellular carcinoma promoted the growth and migration of the cancer cells. Tumour Biol 36: 2077-2085, 2015.

10. Zschiedrich I, Hardeland U, Krones-Herzig A, Berriel Diaz M, Vegiopoulos A, Müggenburg J, Sombroek D, Hofmann TG, Zawatzky R, Yu X, et al: Coactivator function of RIP140 for NFkappaB/RelA-dependent cytokine gene expression. Blood 112: 264-276, 2008.

11. HoPC,Tsui YC,Feng X, Greaves DR and WeiLN: NF-kB-mediated degradation of the co-activator RIP140 regulates inflammatory response and contributes to endotoxin tolerance. Nat Immunol 13 379-386, 2012.

12. Kopanakis K, Tzepi IM, Pistiki A, Carrer DP, Netea MG, Georgitsi M, Lymperi M, Droggiti DI, Liakakos T, Machairas A and Giamarellos-Bourboulis EJ: Pre-treatment with low-dose endotoxin prolongs survival from experimental lethal endotoxic shock: Benefit for lethal peritonitis by Escherichia coli. Cytokine 62: 382-388, 2013
13. Guilherme A, Virbasius JV, Puri V and Czech MP: Adipocyte dysfunctions linking obesity to insulin resistance and type 2 diabetes. Nat Rev Mol Cell Biol 9: 367-377, 2008.

14. Jiang $X$, Huang $L$ and Xing D: Photoactivation of Dok1/ERK/PPAR $\gamma$ signaling axis inhibits excessive lipolysis in insulin-resistant adipocytes. Cell Signal 27: 1265-1275, 2015.

15. Kelley DE, Mokan M, Simoneau JA and Mandarino LJ: Interaction between glucose and free fatty acid metabolism in human skeletal muscle. J Clin Invest 92: 91-98, 1993.

16. Barma P, Bhattacharya S, Bhattacharya A, Kundu R, Dasgupta S, Biswas A, Bhattacharya S, Roy SS and Bhattacharya S: Lipid induced overexpression of NF-kappaB in skeletal muscle cells is linked to insulin resistance. Biochim Biophys Acta 1792: 190-200, 2009

17. Unger RH: Lipotoxic diseases. Annu Rev Med 53: 319-336, 2002.

18. Santomauro AT, Boden G, Silva ME, Rocha DM, Santos RF, Ursich MJ, Strassmann PG and Wajchenberg BL: Overnight lowering of free fatty acids with Acipimox improves insulin resistance and glucose tolerance in obese diabetic and nondiabetic subjects. Diabetes 48: 1836-1841, 1999.

19. Savage DB, Petersen KF and Shulman GI: Disordered lipid metabolism and the pathogenesis of insulin resistance. Physiol Rev 87: 507-520, 2007.

20. Christianson JL, Nicoloro S, Straubhaar J and Czech MP: Stearoyl-CoA desaturase 2 is required for peroxisome proliferator-activated receptor gamma expression and adipogenesis in cultured 3T3-L1 cells. J Biol Chem 283: 2906-2916, 2008.

21. Weisberg SP, McCann D, Desai M, Rosenbaum M, Leibel RL and Ferrante AW Jr: Obesity is associated with macrophage accumulation in adipose tissue. J Clin Invest 112: 1796-1808, 2003.

22. Liu PS, Lin YW, Burton FH and Wei LN: M1-M2 balancing act in white adipose tissue browning-a new role for RIP140. Adipocyte 4: 146-148, 2015

23. Liu PS, Lin YW, Lee B, McCrady-Spitzer SK, Levine JA and Wei LN: Reducing RIP140 expression in macrophage alters ATM infiltration, facilitates white adipose tissue browning, and prevents high-fat diet-induced insulin resistance. Diabetes 63 : 4021-4031, 2014

24. Cancello R, Henegar C, Viguerie N, Taleb S, Poitou C, Rouault C, Coupaye M, Pelloux V, Hugol D, Bouillot JL, et al: Reduction of macrophage infiltration and chemoattractant gene expression changes in white adipose tissue of morbidly obese subjects after surgery-induced weight loss. Diabetes 54: 2277-2786, 2005.

25. Sartipy P and Loskutoff DJ: Monocyte chemoattractant protein 1 in obesity and insulin resistance. Proc Natl Acad Sci USA 100: 7265-7270, 2003

26. Rull A, Camps J, Alonso-Villaverde C and Joven J: Insulin resistance, inflammation, and obesity: Role of monocyte chemoattractant protein-1 (or CCL2) in the regulation of metabolism. Mediators Inflamm 2010: pii: 326580, 2010.

27. Uchida Y, Takeshita K, Yamamoto K, Kikuchi R, Nakayama T, Nomura M, Cheng XW, Egashira K, Matsushita T, Nakamura H and Murohara T: Stress augments insulin resistance and prothrombotic state: Role of visceral adipose-derived monocyte chemoattractant protein-1. Diabetes 61: 1552-1561, 2012.

28. Solinas G, Vilcu C, Neels JG, Bandyopadhyay GK, Luo JL, Naugler W, Grivennikov S, Wynshaw-Boris A, Scadeng M, Olefsky JM and Karin M: JNK1 in hematopoietically derived cells contributes to diet-induced inflammation and insulin resistance without affecting obesity. Cell Metab 6: 386-397, 2007.

29. Tang X, Guilherme A, Chakladar A, Powelka AM, Konda S, Virbasius JV, Nicoloro SM, Straubhaar J and Czech MP: An RNA interference-based screen identifies MAP4K4/NIK as a negative regulator of PPAR gamma, adipogenesis, and insulin-responsive hexose transport. Proc Natl Acad Sci USA 103: 2087-2092, 2006

30. Shulman GI: Cellular mechanisms of insulin resistance. J Clin Invest 106: 171-176, 2000.

31. Tesz GJ, Guilherme A, Guntur KV, Hubbard AC, Tang X, Chawla A and Czech MP: Tumor necrosis factor alpha (TNFalpha) stimulates Map4k4 expression through TNFalpha receptor 1 signaling to c-Jun and activating transcription factor 2. J Biol Chem 282: 19302-19312, 2007

32. Hotamisligil GS, Shargill NS and Spiegelman BM: Adipose expression of tumor necrosis factor-alpha: Direct role in obesity-linked insulin resistance. Science 259: 87-91, 1993.

33. Odegaard JI, Ricardo-Gonzalez RR, Goforth MH, Morel CR, Subramanian V, Mukundan L, Red Eagle A, Vats D, Brombacher F, Ferrante AW and Chawla A: Macrophage-specific PPARgamma controls alternative activation and improves insulin resistance. Nature 447: 1116-1120, 2007. 
34. Yamaguchi Y, Cavallero S, Patterson M, Shen H, Xu J, Kumar SR and Sucov HM: Adipogenesis and epicardial adipose tissue: A novel fate of the epicardium induced by mesenchymal transformation and PPAR $\gamma$ activation. Proc Natl Acad Sci USA 112: 2070-2075, 2015.

35. Imai T, Takakuwa R, Marchand S, Dentz E, Bornert JM, Messaddeq N, Wendling O, Mark M, Desvergne B, Wahli W, et al: Peroxisome proliferator-activated receptor gamma is required in mature white and brown adipocytes for their survival in the mouse. Proc Natl Acad Sci USA 101: 4543-4547, 2004.

36. Loft A, Forss I, Siersbæk MS, Schmidt SF, Larsen AS, Madsen JG, Pisani DF, Nielsen R, Aagaard MM, Mathison A, et al: Browning of human adipocytes requires KLF11 and reprogramming of PPAR $\gamma$ superenhancers. Genes Dev 29: 7-22, 2015.

37. Siersbæk MS, Loft A, Aagaard MM, Nielsen R, Schmidt SF Petrovic N, Nedergaard J and Mandrup S: Genome-wide profiling of peroxisome proliferator-activated receptor $\gamma$ in primary epididymal, inguinal, and brown adipocytes reveals depot-selective binding correlated with gene expression. Mol Cell Biol 32: 3452-3463, 2012.

38. Ait-Lounis A and Laraba-Djebari F: TNF-alpha modulates adipose macrophage polarization to $\mathrm{M} 1$ phenotype in response to scorpion venom. Inflamm Res 64: 929-936, 2015.

39. Bing C: Is interleukin- $1 \beta$ a culprit in macrophage-adipocyte cross talk in obesity? Adipocyte 4: 149-152, 2015.

40. McLaren JE, Michael DR, Ashlin TG and Ramji DP: Cytokines, macrophage lipid metabolism and foam cells: Implications for cardiovascular disease therapy. Prog Lipid Res 50: 331-347, 2011.

41. Qiu Y, Yanase T, Hu H, Tanaka T, Nishi Y, Liu M, Sueishi K, Sawamura $\mathrm{T}$ and Nawata H: Dihydrotestosterone suppresses foam cell formation and attenuates atherosclerosis development. Endocrinology 151: 3307-3316, 2010.

42. Yvan-Charvet L, Ranalletta M, Wang N, Han S, Terasaka N, Li R, Welch C and Tall AR: Combined deficiency of ABCA1 and ABCG1 promotes foam cell accumulation and accelerates atherosclerosis in mice. J Clin Invest 117: 3900-3908, 2007.

43. Wei H, Tarling EJ, McMillen TS, Tang C and LeBoeuf RC: ABCG1 regulates mouse adipose tissue macrophage cholesterol levels and the ratio of M1 to M2 cells during obesity and caloric restriction. J Lipid Res 56: 2337-2347, 2015.

44. Lin YW, Liu PS, Adhikari N, Hall JL and Wei LN: RIP140 contributes to foam cell formation and atherosclerosis by regulating cholesterol homeostasis in macrophages. J Mol Cell Cardiol 79: 287-294, 2015.

45. Dawson MI and Xia Z: The retinoid X receptors and their ligands. Biochim Biophys Acta 1821: 21-56, 2012.

46. Calkin AC and Tontonoz P: Liver X receptor signaling pathways and atherosclerosis. Arterioscler Thromb Vasc Biol 30 $1513-1518,2010$

47. He Y, Zhang L, Li Z, Gao H, Yue Z, Liu Z, Liu X, Feng X and Liu P: RIP140 triggers foam-cell formation by repressing ABCA1/G1 expression and cholesterol efflux via liver X receptor. FEBS Lett 589: 455-460, 2015
48. Calkin AC and Tontonoz P: Transcriptional integration of metabolism by the nuclear sterol-activated receptors $1 X R$ and FXR. Nat Rev Mol Cell Biol 13: 213-224, 2012

49. Ho PC, Chang KC, Chuang YS and Wei LN: Cholesterol regulation of receptor-interacting protein 140 via microRNA-33 in inflammatory cytokine production. FASEB J 25: 1758-1766, 2011.

50. Rayner KJ, Sheedy FJ, Esau CC, Hussain FN, Temel RE, Parathath S, van Gils JM, Rayner AJ, Chang AN, Suarez Y, et al: Antagonism of mir-33 in mice promotes reverse cholesterol transport and regression of atherosclerosis. J Clin Invest 121: 2921-2931, 2011.

51. Dávalos A and Fernández-Hernando C: From evolution to revolution: miRNAs as pharmacological targets for modulating cholesterol efflux and reverse cholesterol transport. Pharmacol Res 75: 60-72, 2013.

52. Karasawa T and Takahashi M: RIP140 as a novel therapeutic target in the treatment of atherosclerosis. J Mol Cell Cardiol 81: $136-138,2015$

53. Jawad I, Lukšić I and Rafnsson SB: Assessing available information on the burden of sepsis: Global estimates of incidence, prevalence and mortality. J Glob Health 2: 010404, 2012.

54. Charchaflieh J, Wei J, Labaze G, Hou YJ, Babarsh B, Stutz H, Lee H, Worah S and Zhang M: The role of complement system in septic shock. Clin Dev Immunol 2012: 407324, 2012.

55. Wang X and Quinn PJ: Lipopolysaccharide: Biosynthetic pathway and structure modification. Prog Lipid Res 49: 97-107, 2010.

56. Xiong Y, Pennini M, Vogel SN and Medvedev AE: IRAK4 kinase activity is not required for induction of endotoxin tolerance but contributes to TLR2-mediated tolerance. J Leukoc Biol 94: 291-300, 2013.

57. Laird MH, Rhee SH, Perkins DJ, Medvedev AE, Piao W, Fenton MJ and Vogel SN: TLR4/MyD88/PI3K interactions regulate TLR4 signaling. J Leukoc Biol 85: 966-977, 2009.

58. Nahid MA, Satoh M and Chan EK: MicroRNA in TLR signaling and endotoxin tolerance. Cell Mol Immunol 8: 388-403, 2011.

59. Liew FY, Xu D, Brint EK and O'Neill LA: Negative regulation of Toll-like receptor-mediated immune responses. Nat Rev Immunol 5: 446-458, 2005.

60. Park SH, Park-Min KH, Chen J, Hu X and Ivashkiv LB: Tumor necrosis factor induces GSK 3 kinase-mediated cross-tolerance to endotoxin in macrophages. Nat Immunol 12: 607-615, 2011

61. Chen $\mathbf{J}$ and Ivashkiv LB: IFN $-\gamma$ abrogates endotoxin tolerance by facilitating Toll-like receptor induced chromatin remodeling. Proc Natl Acad Sci USA 107: 19438-19443, 2010.

62. Chen Y, Liu Z, Liang S, Luan X, Long F, Chen J, Peng Y, Yan L and Gong J: Role of Kupffer cells in the induction of tolerance of orthotopic liver transplantation in rats. Liver Transpl 14: 823-836, 2008 